\title{
Interpolação Espacial da Precipitação no Estado do Espírito Santo
}

\author{
Kennedy Ribeiro da Silva1 ${ }^{1}$, Roberto Avelino Cecílioº ${ }^{2}$ Alexandre Cândido Xavier ${ }^{3}$, \\ José Ricardo Macedo Pezzopane ${ }^{4}$, Giovanni de Oliveira Garcia ${ }^{3}$
}

\author{
${ }^{1}$ Faculdades Integradas de Ensino Superior Linhares - FACELI \\ ${ }^{2}$ Departamento de Engenharia Florestal, Universidade Federal do Espírito Santo - UFES \\ ${ }^{3}$ Departamento de Engenharia Rural, Universidade Federal do Espírito Santo - UFES \\ ${ }^{4}$ EMBRAPA - Pecuária Sudeste
}

\begin{abstract}
RESUMO
O conhecimento da variabilidade espacial da precipitação é importante em diversos ramos das ciências agrárias e ambientais, como, por exemplo, na produção das culturas, no manejo dos recursos hídricos, na avaliação ambiental e em estudos de erosão hídrica. O presente estudo teve como objetivo avaliar dois métodos de interpolação para a espacialização da precipitação mensal no Estado do Espírito Santo. Utilizaram-se dados de precipitação mensal, compreendidos no período de 1977 a 2006, de 110 postos pluviométricos, sendo 94 do Espírito Santo e 16 de Estados vizinhos. Avaliaram-se os métodos de interpolação Inverso da Potência da Distância (IPD), considerando-se as potências de 2 até 6 e a Krigagem (KR), modelos exponencial, esférico e linear, por meio da validação cruzada. A avaliação e a seleção do melhor método foram feitas a partir de índices estatísticos (índice de confiança, coeficiente de eficiência ajustado e erro médio percentual). Os resultados mostram que o método de KR é mais eficiente para a interpolação espacial da precipitação mensal no Espírito Santo, em comparação ao método IPD, e apresenta erros médios percentuais entre 7,9 e 14,6\%.
\end{abstract}

Palavras-chave: geoestatística, séries temporais, dependência espacial.

\section{Rainfall Spatial Interpolation in the State of the Espirito Santo, Brazil}

\begin{abstract}
The knowledge of spatial variability of rainfall is important in many agricultural and environmental sciences applications, such as crop production, water resources management, environmental management, and soil erosion studies. The purpose of this study was to evaluated two spatial interpolation methods applied to monthly rainfall in the State Espirito Santo, Brazil. Thirty years of monthly rainfall data (1997-2006) from 110 rain gauge stations, 94 stations in the State of Esprito Santo and 16 stations in the neighboring states, were used. The spatial interpolation methods 'Inverse Distance to a Power' (IPD), considering power from 2 to 6, and 'Kriging' (KR), exponential, spherical and linear models, were evaluated through the use of cross validation. The evaluation and selection of the best spatial interpolation methods was done by analyzing the statistical indexes (trusty index, adjusted efficiency coefficient and mean percent error). Results showed that KR method had better performance than IPD method concerning spatial interpolation of monthly rainfall in the State of Espirito Santo, presenting mean percent errors varying from 7.9 to $14.6 \%$.
\end{abstract}

Keywords: geostatistics, time series, spatial dependence. 


\section{INTRODUÇÃO}

O conhecimento da precipitação pluvial é importante em ramos das ciências agrárias e ambientais, como, por exemplo, na estimativa de produção das culturas, no manejo dos recursos hídricos, na avaliação ambiental, na conservação dos solos, no zoneamento agroclimático e no potencial de desenvolvimento de patógenos, dentre outros. O conhecimento de sua distribuição espacial é fundamental para aplicações em modelagem hidrológica de bacias hidrográficas, transporte de poluentes, dinâmica de rios e estimativa de perdas de solo (Fabry, 2004; Tao et al., 2009).

No Estado do Espírito Santo, como na maior parte do território brasileiro, a disponibilidade de informações meteorológicas, principalmente da precipitação pluvial, ainda mostra-se deficiente, o que se deve à baixa densidade e à má distribuição dos postos pluviométricos e/ou pluviográficos. Para contornar esses problemas, diversos pesquisadores sugerem a utilização de técnicas de interpolação espacial para estimar e espacializar os totais precipitados (Barbosa et al., 2005; Barbosa, 2006; Carvalho \& Assad, 2005; Vieira et al., 2010).

Existem várias metodologias para interpolar dados espaciais, sendo as mais comumente utilizadas, segundo a literatura, o "Inverso da Potência da Distância-IPD” e a "Krigagem-KR". Na escolha do método de interpolação a ser utilizado, geralmente, considera-se apenas um critério de escolha, aquele com o qual o usuário tem maior afinidade (Xavier et al., 2010). Até pouco tempo atrás, não se levava em consideração a preocupação com a qualidade da estimativa, o que somente vem ocorrendo recentemente (Price et al., 2000; Teegavarapu \& Chandramouli, 2005; Amorim et al., 2008; Lu \& Wong, 2008; Castro et al., 2010).

A qualidade da interpolação espacial de dados climáticos depende da densidade e da distribuição dos pontos usados no cálculo, além da existência de correlação entre os modelos estatísticos dos interpoladores e os fenômenos em estudo. A escolha de um modelo de interpolação apropriado é essencial para se obterem mapas de espacialização confiáveis. Diante desse fato, é importante avaliar o desempenho dos interpoladores para cada variável estudada, o que pode ser feito com a utilização de índices estatísticos apropriados (Castro et al., 2010; Falivene et al., 2010).

A partir do exposto, o presente trabalho teve por objetivo avaliar dois métodos de interpolação destinados à espacialização da precipitação mensal no Estado do Espírito Santo.

\section{MATERIAL E MÉTODOS}

A área de estudo compreende o Estado do Espírito Santo, localizado entre as latitudes $17^{\circ} 52^{\prime}$ e $21^{\circ} 19^{\prime} \mathrm{S}$ e as longitudes $39^{\circ} 38^{\prime}$ e $41^{\circ} 50^{\prime} \mathrm{O}$, na região Sudeste do Brasil. Na região, as precipitações são notadamente influenciadas pela maritimidade/ continentalidade, pelo relevo (picos elevados, depressões intermontanhas, regiões montanhosas e baixadas litorâneas) e pelas linhas de instabilidade, principalmente no verão. No inverno, há predominância de atuação dos sistemas frontais. $\mathrm{Na}$ região, ainda atuam sistemas atmosféricos de grande escala, como Anticiclone Subtropical do Atlântico Sul (ASAS), Baixa de Chaco, Alta da Bolívia, Alta Polar e Corrente de Jato (Vianello \& Alves, 2000).

A topografia do Espírito Santo é formada por áreas planas no litoral e área montanhosa no interior (Figura 1). De acordo com Siqueira et al. (2004), o clima é influenciado, de maneira marcante, pela topografia e pela exposição das encostas, condicionadoras de chuvas orográficas, associadas à proximidade do oceano. Baseado no sistema de classificação climática de Köppen, a região apresenta as zonas climáticas A e C, sendo classificadas como climas úmidos. No Estado, são encontrados os seguintes subtipos climáticos: Aw, Am, Cf e Cw, e as variações Cfa, Cfb, Cwa e Cwb (Siqueira et al., 2004).

Utilizaram-se séries históricas mensais de precipitação do período de 1977 a 2006 (30 anos), obtidas de 110 postos pluviométricos (Figura 2). Onze são pertencentes à rede de postos do Instituto Capixaba de Pesquisas e Extensão Rural (INCAPER), três do Instituto Nacional de Meteorologia (INMET) e 96 da Agência Nacional de Águas (ANA), localizados no Estado do Espírito Santo e em Estados vizinhos (Rio de Janeiro, Minas Gerais e Bahia), tendo como objetivo minimizar o efeito de borda no processo de interpolação. 


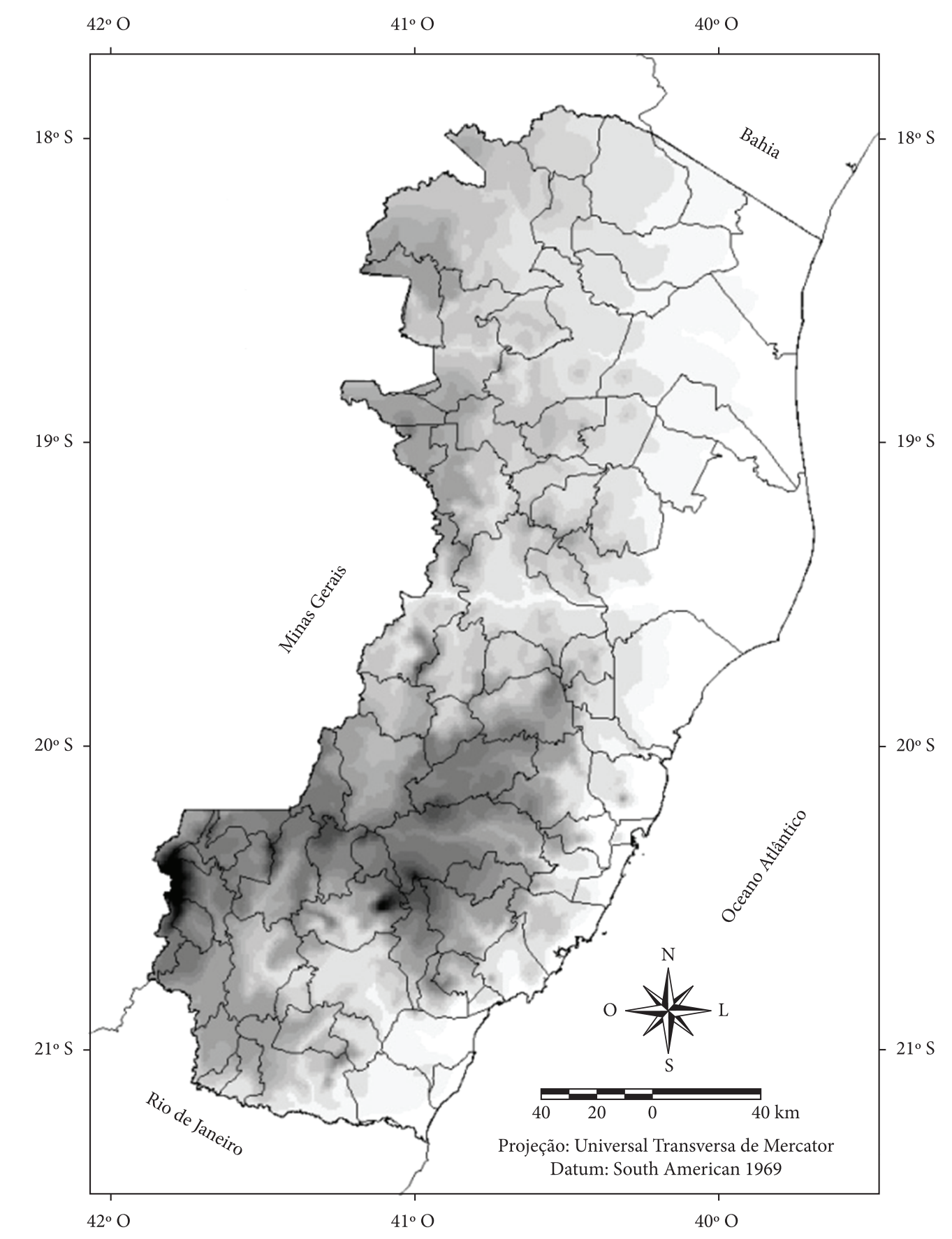

\begin{tabular}{|c|c|c|c|c|}
\hline$<48 \mathrm{~m}$ & $48-100 \mathrm{~m}$ & $100-200 \mathrm{~m}$ & $200-300 \mathrm{~m}$ & $300-400 \mathrm{~m}$ \\
\hline $400-500 \mathrm{~m}$ & $500-600 \mathrm{~m}$ & $600-700 \mathrm{~m}$ & $700-800 \mathrm{~m}$ & $800-900 \mathrm{~m}$ \\
\hline $900-1.000 \mathrm{~m}$ & $1.000-1.100 \mathrm{~m}$ & $1.100-1.200 \mathrm{~m}$ & $1.200-1.300 \mathrm{~m}$ & $1.300-1.400 \mathrm{~m}$ \\
\hline $1.400-1.500 \mathrm{~m}$ & $1.500-1.600 \mathrm{~m}$ & $1.600-1.700 \mathrm{~m}$ & $1.700-1.800 \mathrm{~m}$ & $>1.800 \mathrm{~m}$ \\
\hline
\end{tabular}

Figura 1. Mapa altimétrico do Estado do Espírito Santo.

Figure 1. Altimetric map of the State of Espírito Santo. 
$40^{\circ} \mathrm{O}$

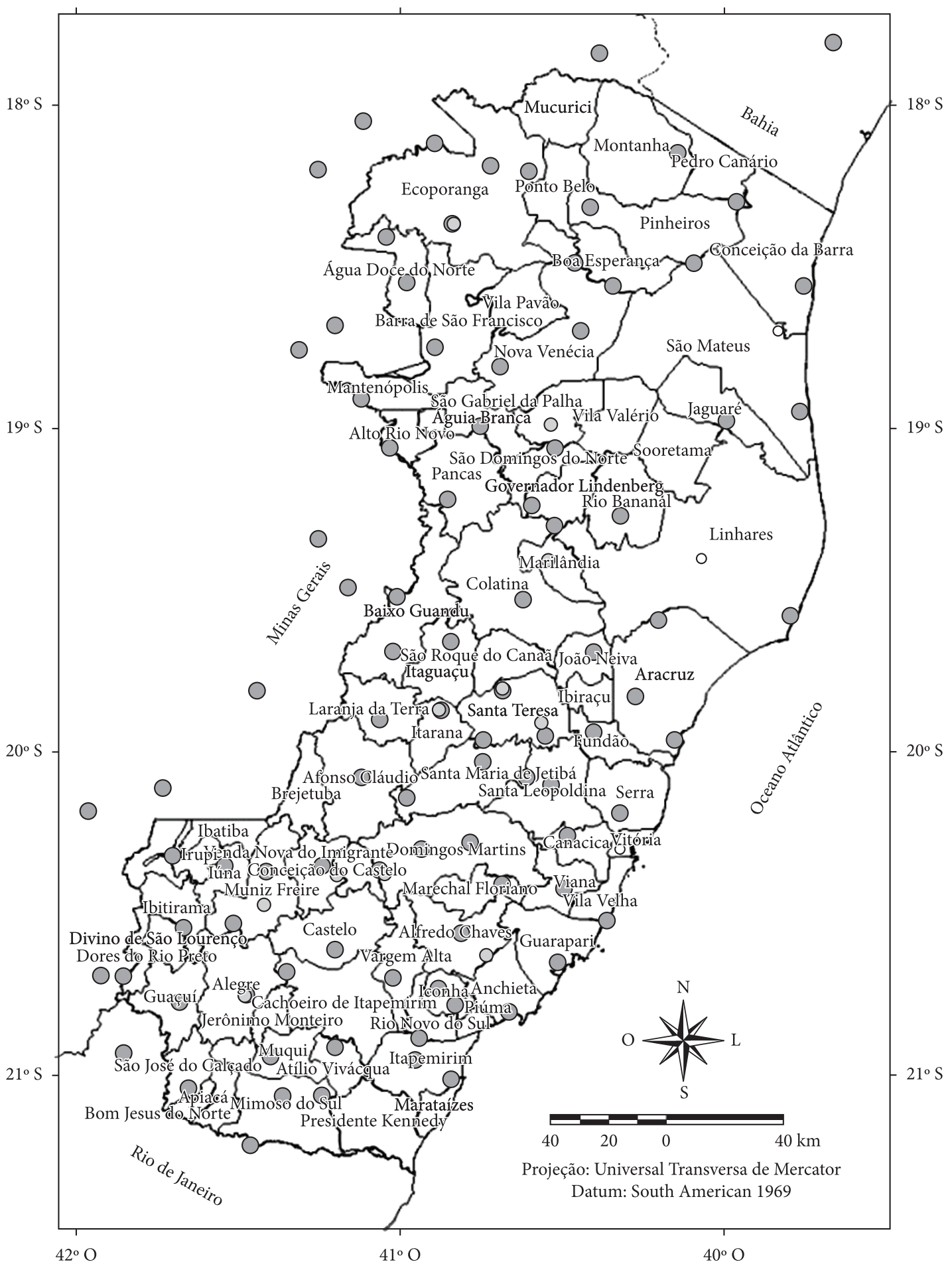

ANA O INCAPER O INMET

Figura 2. Localização dos postos pluviométricos utilizados no Estado do Espírito Santo e em Estados vizinhos. Figure 2. Spatial location of the raingauge stations used on the State of Espírito Santo and neighborhood States. 
Para a espacialização das precipitações mensais, avaliaram-se os seguintes métodos de interpolação neste estudo: IPD, considerando-se as potências de 2 a 6 (avaliadas em outros trabalhos da literatura, como Castro et al. (2010)), e KR ordinária, considerando-se os modelos de semivariograma experimental linear (KRLIN), esférico (KRESF) e exponencial (KREXP).

A interpolação pelo IPD considera que o valor da variável a ser estimado numa posição qualquer é calculado pelos " $n$ " vizinhos mais próximos ponderados pelo inverso da sua distância elevada a uma potência, conforme a Equação 1 (Caruso \& Quarta, 1998):

$$
z_{i}=\frac{\sum_{j=1}^{n}\left(\frac{z_{j}}{d_{i j}^{k}}\right)}{\sum_{j=1}^{n}\left(\frac{1}{d_{i j}^{k}}\right)}
$$

em que: $z_{i}=$ precipitação interpolada no ponto $i$; $\mathrm{d}_{\mathrm{ij}}=$ distância entre os pontos i e $\mathrm{j} ; \mathrm{z}_{\mathrm{j}}=$ precipitação medida no ponto $\mathrm{j} ; \mathrm{n}=$ número de postos usados na interpolação; $\mathrm{k}$ = potência utilizada (variou entre 2 e 6 no presente trabalho).

Para a aplicação do método KR ordinária, primeiramente realizou-se a análise estatística exploratória dos dados, a fim de se verificar alguma anormalidade dos dados de chuva. Logo em seguida, procedeu-se à análise geoestatística, visando quantificar o grau de dependência espacial dos dados, por meio de um semivariograma experimental, estimado pela Equação 2, de acordo com a metodologia descrita por Vieira (2000):

$$
\hat{\gamma}(\mathbf{h})=\frac{1}{2 N(h)} \sum_{i=1}^{N(h)}\left\{z\left(\mathbf{x}_{i}\right)-z\left(\mathbf{x}_{i}+\mathbf{h}\right)\right\}^{2}
$$

em que: $\hat{y}(\mathrm{~h})=$ semivariância estimada para uma distância $h ; N(h)=$ número de pares amostrais do atributo $\mathrm{z}$ (precipitação) separados por uma distância $h ; \mathrm{x}_{\mathrm{i}}$ ex $+\mathrm{h}=$ locais de amostragens separados por uma distância $\mathrm{h} ; \mathrm{z}\left(\mathrm{x}_{\mathrm{i}}\right)$ e $\mathrm{z}\left(\mathrm{x}_{\mathrm{i}}+\mathrm{h}\right)=$ valores de precipitação medidos nos locais correspondentes.

$\mathrm{O}$ ajuste dos parâmetros dos semivariogramas (efeito pepita- $c_{0}$; variância estrutural- $c_{1}$; e alcance-a) foi realizado por meio da técnica de validação cruzada (Vieira, 2000), sendo avaliados os modelos linear, esférico e exponencial, descritos pelas Equações 3-5, respectivamente.

$\hat{\gamma}(\mathbf{h})=c_{0}+\frac{c_{1}}{a} \mathbf{h}$

$\hat{\gamma}(\mathbf{h})=c_{0}+c_{1}\left\{\frac{3 \mathbf{h}}{2 a}-1 / 2(\mathbf{h} / a)^{3}\right\}$

$\hat{\gamma}(\mathbf{h})=c_{0}+c_{1}\{1-\exp (-\mathbf{h} / a)\}$

A verificação da acurácia dos interpoladores para espacializar a precipitação mensal foi realizada por meio da validação cruzada (Robinson \& Metternicht, 2006). Para sua aplicação, é necessário que um ponto (posto pluviométrico) seja extraído, pois assim é possível obter o valor estimado (E) referente ao ponto retirado e posteriormente compará-lo com o valor observado $(\mathrm{O})$ da variável. Esse procedimento foi realizado para cada um dos 110 postos pluviométricos com auxílio da rotina computacional desenvolvida em ambiente MatLab (MathWorks, 2000), por Xavier et al. (2010).

A avaliação e a seleção dos interpoladores foram feitas a partir do cálculo dos índices estatísticos: índice de confiança (c) (Camargo \& Sentelhas, 1997), coeficiente de eficiência ajustado (E') (Legates \& McCabe Junior, 1999) e Erro Médio Percentual (EMP), conforme as equações a seguir (Equações 6-8):

$c=r\left(1-\frac{\sum_{i=1}^{J}\left(O_{i}-E_{i}\right)^{2}}{\sum_{i=1}^{J}\left(\left|E_{i}-\bar{O}\right|+\left|O_{i}-\bar{O}\right|\right)^{2}}\right)$

$E^{\prime}=1-\frac{\sum_{i=1}^{J}\left|O_{i}-E_{i}\right|}{\sum_{i=1}^{J}\left|O_{i}-\bar{O}\right|}$

$E M P=\frac{\sum_{i=1}^{J}\left(\frac{\left|O_{i}-E_{i}\right|}{O_{i}}\right)}{J} \cdot 100$

em que: $r$ = coeficiente de determinação entre valores observados e estimados; J = número de observações; $\mathrm{O}=$ valor observado experimentalmente; $\mathrm{E}=$ valor estimado pelo método; $\overline{\mathrm{O}}=$ média dos valores observados. 
O índice (c) permite analisar conjuntamente a precisão e a exatidão dos resultados por meio de uma escala de desempenho (Tabela 1).

A precipitação média anual estimada para cada posto pluviométrico foi obtida pela soma das precipitações mensais estimadas pelos interpoladores de melhor desempenho (determinados anteriormente). Posteriormente, o desempenho dessa forma de estimativa da precipitação anual foi calculado por meio dos índices estatísticos c, E’ e EMP.

\section{RESULTADOS E DISCUSSÃO}

$\mathrm{Na}$ Tabela 2, verifica-se que os meses com maiores precipitações foram novembro, dezembro e janeiro, característica marcante da região Sudeste brasileira, de acordo com Vianello \& Alves (2000), e que ocorre muito em função da atuação da Zona de

Tabela 1. Critérios para análise de desempenho do modelo com base no índice de confiança (c) (Camargo \& Sentelhas, 1997).

Table 1. Criteria to model performance analysis based on trusty index (c) (Camargo \& Sentelhas, 1997).

\begin{tabular}{cc|}
\hline Valor de c & Desempenho \\
\hline$>0,85$ & Ótimo \\
\hline 0,76 a 0,85 & Muito bom \\
\hline 0,66 a 0,75 & Bom \\
0,61 a 0,65 & Mediano \\
\hline 0,51 a 0,60 & Sofrível \\
0,41 a 0,50 & Mau \\
$\leq 0,40$ & Péssimo \\
\hline
\end{tabular}

Convergência do Atlântico Sul e da intensa atividade convectiva (Quadro et al., 1996). Os meses com menores precipitações foram aqueles entre junho e agosto, período marcado pela influência das frentes frias de intensidade fraca (Moraes, 2004). Nos meses de menores precipitações, foram evidenciados os maiores coeficientes de variação $(\mathrm{CV})$. É interessante ressaltar que os valores de CV são baixos (entre 14 e $54 \%)$, comparativamente aos encontrados na região Nordeste (entre 45 e 312\%) por Silva et al. (2011), e semelhantes aos encontrados por Viola et al. (2010) (entre $17 \%$ e 60\%), para Minas Gerais, Estado vizinho.

$\mathrm{Na}$ Tabela 3, observa-se, por meio dos índices estatísticos, que o interpolador $\mathrm{KR}$ ordinária, utilizando-se o modelo KRLIN, apresentou melhor desempenho para a estimativa e a espacialização das precipitações mensais no Estado do Espírito Santo. A exceção foi o mês de junho, no qual o modelo KREXP mostrou melhor desempenho.

Os valores de EMP dos melhores interpoladores foram baixos (inferiores a 14,6\%). Esses valores são aceitáveis em se tratando de uma variável meteorológica com variabilidade espacial tão alta quanto a precipitação (Silva et al., 2003). Para as precipitações de cada mês e para a média anual, os melhores métodos de interpolação apresentaram valores do coeficiente E' variando entre 0,46 a 0,90, indicando bom desempenho. Esse índice é significativo, variando entre $-\infty$ e 1 , sendo os valores negativos indicativos de desempenho insatisfatório, enquanto os maiores valores correspondem aos

Tabela 2. Estatística descritiva da precipitação mensal no Espírito Santo no período de 1977-2006.

Table 2. Monthly rainfall descriptive statistics for Espírito Santo State (1977-2006).

\begin{tabular}{|c|c|c|c|c|}
\hline Mês & Média (mm) & Mínimo (mm) & Máximo (mm) & Coef. de variação (CV) (\%) \\
\hline Janeiro & 184,6 & 101,5 & 291,5 & 19 \\
\hline Fevereiro & 102,8 & 55,2 & 143,9 & 19 \\
\hline Março & 144,3 & 83,5 & 231,7 & 19 \\
\hline Abril & 88,6 & 44,2 & 167,1 & 30 \\
\hline Maio & 49,9 & 26,2 & 106,9 & 31 \\
\hline Junho & 32,6 & 10,2 & 78,1 & 45 \\
\hline Julho & 38,1 & 6,6 & 92,2 & 54 \\
\hline Agosto & 38,1 & 16,2 & 93,3 & 40 \\
\hline Setembro & 59,0 & 25,1 & 124,6 & 39 \\
\hline Outubro & 101,1 & 60,8 & 161,1 & 19 \\
\hline Novembro & 195,3 & 147,7 & 277,7 & 14 \\
\hline Dezembro & 223,0 & 128,7 & 346,8 & 17 \\
\hline
\end{tabular}


Tabela 3. Índices estatísticos para análise do desempenho dos interpoladores para estimativa da precipitação média mensal no Estado do Espírito Santo.

Table 3. Statistic indexes used to analyze monthly and annual rainfall interpolators at Espírito Santo.

\begin{tabular}{|c|c|c|c|c|c|c|c|c|c|}
\hline \multirow{2}{*}{ Mês } & \multirow{2}{*}{ Îndice } & \multicolumn{8}{|c|}{ Interpolador } \\
\hline & & IPD2 & IPD3 & IPD4 & IPD5 & IPD6 & KREXP & KRESF & KRLIN \\
\hline \multirow{4}{*}{ Jan. } & c & 0,61 & 0,60 & 0,58 & 0,57 & 0,56 & 0,75 & 0,70 & 0,78 \\
\hline & Des. & Mediano & Sofrível & Sofrível & Sofrível & Sofrível & Bom & Bom & Muito bom \\
\hline & $\mathrm{E}^{\prime}$ & 0,50 & 0,48 & 0,45 & 0,43 & 0,41 & 0,69 & 0,63 & 0,73 \\
\hline & EMP & 11,4 & 11,7 & 12,1 & 12,3 & 12,6 & 8,8 & 9,2 & 8,1 \\
\hline \multirow{4}{*}{ Fev. } & c & 0,56 & 0,53 & 0,52 & 0,50 & 0,49 & \multirow{4}{*}{$\begin{array}{l}\text { Não houve } \\
\text { ajuste }\end{array}$} & 0,68 & 0,74 \\
\hline & Des. & Sofrível & Sofrível & Sofrível & Mau & Mau & & Bom & Bom \\
\hline & $\mathrm{E}^{\prime}$ & 0,44 & 0,41 & 0,38 & 0,34 & 0,31 & & 0,60 & 0,68 \\
\hline & EMP & 12,2 & 12,5 & 12,8 & 13,1 & 13,3 & & 10,0 & 8,7 \\
\hline \multirow{4}{*}{ Mar. } & c & 0,39 & 0,38 & 0,37 & 0,35 & 0,34 & \multirow{4}{*}{$\begin{array}{l}\text { Não houve } \\
\text { ajuste }\end{array}$} & 0,64 & 0,67 \\
\hline & Des. & Péssimo & Péssimo & Péssimo & Péssimo & Péssimo & & Mediano & Bom \\
\hline & E’ & 0,24 & 0,22 & 0,19 & 0,17 & 0,14 & & 0,56 & 0,60 \\
\hline & EMP & 12,8 & 13,1 & 13,4 & 13,7 & 14,0 & & 9,9 & 9,1 \\
\hline \multirow{4}{*}{ Abr. } & c & 0,64 & 0,65 & 0,65 & 0,65 & 0,65 & \multirow{4}{*}{$\begin{array}{l}\text { Não houve } \\
\text { ajuste }\end{array}$} & 0,81 & 0,82 \\
\hline & Des. & Mediano & Mediano & Mediano & Mediano & Mediano & & Muito bom & Muito bom \\
\hline & $E^{\prime}$ & 0,55 & 0,55 & 0,54 & 0,54 & 0,53 & & 0,76 & 0,77 \\
\hline & EMP & 14,7 & 15,1 & 15,3 & 15,5 & 15,6 & & 11,5 & 10,7 \\
\hline \multirow{4}{*}{ Maio } & c & 0,65 & 0,65 & 0,64 & 0,63 & 0,63 & 0,75 & 0,71 & 0,79 \\
\hline & Des. & Mediano & Mediano & Mediano & Mediano & Mediano & Bom & Bom & Muito bom \\
\hline & $\mathrm{E}^{\prime}$ & 0,57 & 0,56 & 0,55 & 0,53 & 0,52 & 0,69 & 0,63 & 0,74 \\
\hline & EMP & 13,9 & 13,9 & 14,0 & 14,3 & 14,6 & 11,9 & 13,2 & 11,3 \\
\hline \multirow{4}{*}{ Jun. } & c & 0,72 & 0,71 & 0,70 & 0,69 & 0,68 & 0,91 & 0,87 & 0,90 \\
\hline & Des. & Bom & Bom & Bom & Bom & Bom & Ótimo & Ótimo & Ótimo \\
\hline & $E^{\prime}$ & 0,67 & 0,65 & 0,64 & 0,62 & 0,61 & 0,89 & 0,83 & 0,87 \\
\hline & EMP & 18,7 & 18,8 & 19,1 & 19,5 & 20,0 & 12,0 & 15,1 & 12,6 \\
\hline \multirow{4}{*}{ Jul. } & c & 0,75 & 0,74 & 0,73 & 0,72 & 0,71 & \multirow{4}{*}{$\begin{array}{l}\text { Não houve } \\
\text { ajuste }\end{array}$} & 0,88 & 0,93 \\
\hline & Des. & Bom & Bom & Bom & Bom & Bom & & Ótimo & Ótimo \\
\hline & $\mathrm{E}^{\prime}$ & 0,69 & 0,68 & 0,66 & 0,64 & 0,63 & & 0,83 & 0,90 \\
\hline & EMP & 26,2 & 26,0 & 25,9 & 26,0 & 26,2 & & 19,8 & 14,6 \\
\hline \multirow{4}{*}{ Ago. } & c & 0,63 & 0,63 & 0,62 & 0,61 & 0,60 & \multirow{4}{*}{$\begin{array}{l}\text { Não houve } \\
\text { ajuste }\end{array}$} & 0,86 & 0,88 \\
\hline & Des. & Mediano & Mediano & Mediano & Mediano & Sofrível & & Ótimo & Ótimo \\
\hline & $E^{\prime}$ & 0,54 & 0,52 & 0,49 & 0,47 & 0,45 & & 0,79 & 0,85 \\
\hline & EMP & 21,1 & 20,8 & 20,8 & 21,1 & 21,3 & & 15,5 & 12,5 \\
\hline \multirow{4}{*}{ Set. } & c & 0,74 & 0,73 & 0,73 & 0,72 & 0,72 & \multirow{4}{*}{$\begin{array}{l}\text { Não houve } \\
\text { ajuste }\end{array}$} & 0,86 & 0,86 \\
\hline & Des. & Bom & Bom & Bom & Bom & Bom & & Ótimo & Ótimo \\
\hline & $\mathrm{E}^{\prime}$ & 0,66 & 0,66 & 0,64 & 0,62 & 0,60 & & 0,81 & 0,82 \\
\hline & EMP & 17,9 & 18,0 & 18,0 & 18,1 & 18,2 & & 13,0 & 12,0 \\
\hline \multirow{4}{*}{ Out. } & c & 0,52 & 0,50 & 0,50 & 0,51 & 0,50 & 0,59 & 0,60 & 0,61 \\
\hline & Des. & Sofrível & Sofrível & Sofrível & Sofrível & Sofrível & Sofrível & Sofrível & Mediano \\
\hline & $\mathrm{E}^{\prime}$ & 0,39 & 0,37 & 0,35 & 0,32 & 0,30 & 0,49 & 0,49 & 0,50 \\
\hline & EMP & 11,9 & 12,0 & 12,2 & 12,4 & 12,6 & 9,8 & 9,9 & 9,8 \\
\hline & c & 0,34 & 0,33 & 0,32 & 0,31 & 0,30 & 0,55 & 0,56 & 0,56 \\
\hline Nov & Des. & Péssimo & Péssimo & Péssimo & Péssimo & Péssimo & Sofrível & Sofrível & Sofrível \\
\hline & $E^{\prime}$ & 0,12 & 0,08 & 0,04 & 0,01 & $-0,02$ & 0,46 & 0,46 & 0,46 \\
\hline & EMP & 9,8 & 10,1 & 10,3 & 10,6 & 10,8 & 8,0 & 8,0 & 7,9 \\
\hline & c & 0,33 & 0,33 & 0,34 & 0,34 & 0,34 & 0,64 & 0,63 & 0,68 \\
\hline Dez & Des. & Péssimo & Péssimo & Péssimo & Péssimo & Péssimo & Mediano & Mediano & Bom \\
\hline Dez. & E' & 0,20 & 0,17 & 0,15 & 0,12 & 0,10 & 0,57 & 0,56 & 0,61 \\
\hline & EMP & 12,1 & 12,1 & 12,1 & 12,2 & 12,3 & 8,4 & 8,4 & 8,0 \\
\hline
\end{tabular}

c: índice de confiança; Des.: desempenho conforme índice c; E’: coeficiente de eficiência; EMP: erro médio percentual. 
melhores desempenhos, segundo Legates \& McCabe Junior. (1999). O índice (c) foi, na maioria das vezes, satisfatório (Bom, Muito bom ou Ótimo), com exceção feita aos meses de outubro e novembro, nos quais as classificações foram Medianas e Sofríveis, respectivamente.

Os meses de outubro e novembro apresentaram, para todos os interpoladores, os piores desempenhos medidos por meio dos índices c e E', o que se deve à grande variabilidade espacial da precipitação justamente nesses meses, em que as partes Norte e Noroeste do Espírito Santo se encontram no período seco, enquanto outras se encontram no período chuvoso; tal fato ocorre por existirem, no Estado, regiões pluviométricas de características distintas (Keller Filho et al., 2005; Melo Júnior et al., 2006) com relação, principalmente, ao início da estação chuvosa, conforme descrito por Minuzzi et al. (2007).

Os melhores desempenhos de interpoladores foram nos meses de junho a setembro, com os maiores valores de E' $(0,82$ a 0,9$)$ e as melhores classificações conforme índice c (Ótimo). Esses são meses mais secos no Estado do Espírito Santo, segundo Blain et al. (2010), em que os valores de CV foram maiores (Tabela 2). O melhor desempenho do método KRordinária, em comparação ao método IPD para estimativa e espacialização das precipitações, foi constatado por outros pesquisadores, como, por exemplo, Siqueira et al. (2007), em Minas Gerais, e Baú et al. (2006), no Paraná.
A superioridade da KR ordinária pode ser explicada pelo fato de que esta, segundo Akkala et al. (2010), é indicada para a aplicação em regiões onde a distribuição espacial dos dados observados é boa e sem descontinuidades significativas, enquanto o IPD é indicado para locais com distribuição espacial boa, além de alta densidade de postos (que não é o caso do presente estudo). Além disso, cabe salientar que, para o interpolador KR, a partir dos dados de entrada, se determinam os melhores parâmetros para modelagem da função matemática do semivariograma experimental, resultando em boas estimativas quando os dados são representativos da região (Miranda et al., 2009; Sivapragasam et al., 2010).

Com relação à espacialização das precipitações médias anuais, os valores dos coeficientes estatísticos c, E’ e EMP foram de 0,67 (Bom), 0,60 e $7,4 \%$, respectivamente. No trabalho de Castro et al. (2010), que utilizaram a mesma base de dados de precipitação deste estudo, o método KR ordinária com o modelo de semivariograma exponencial apresentou melhores resultados, com índice $\mathrm{c}$ igual a 0,66 (Bom) e E' igual a 0,40, sendo inferiores aos obtidos neste estudo para a espacialização da precipitação média anual. Demonstra-se, assim, que a espacialização das precipitações anuais por meio da soma das espacializações das precipitações mensais apresenta melhor resultado, embora semelhante ao da Krigagem.

Tabela 4. Parâmetros dos modelos de semivariograma ajustados para os modelos de Krigagem ordinária para estimativa espacial das precipitações mensais no Espírito Santo.

Table 4. Semivariogram model parameters adjusted to Ordinary Kriging Models applied to predict the spatial distribution of monthly rainfall for the State of Espírito Santo.

\begin{tabular}{|c|c|c|c|c|c|}
\hline Mês & Modelo & $\begin{array}{c}\text { Efeito } \\
\text { pepita-c }_{0}\end{array}$ & $\begin{array}{c}\text { Variância } \\
\text { estrutural-c }\end{array}$ & $\begin{array}{c}\text { Alcance-a } \\
\left({ }^{\circ}\right)\end{array}$ & $\begin{array}{c}\text { Alcance } \\
(\mathbf{k m})\end{array}$ \\
\hline Janeiro & Linear & 285,57 & 1096,30 & 1,00 & 111 \\
\hline Fevereiro & Linear & 108,66 & 388,80 & 1,00 & 111 \\
\hline Março & Linear & 282,68 & 594,11 & 1,00 & 111 \\
\hline Abril & Linear & 127,04 & 742,48 & 1,00 & 111 \\
\hline Maio & Linear & 35,96 & 217,35 & 1,00 & 111 \\
\hline Junho & Exponencial & 0,00 & 219,11 & 0,49 & 54 \\
\hline Julho & Linear & 54,17 & 468,86 & 1,00 & 111 \\
\hline Agosto & Linear & 77,67 & 218,12 & 1,00 & 111 \\
\hline Setembro & Linear & 154,92 & 453,38 & 1,00 & 111 \\
\hline Outubro & Linear & 126,97 & 204,90 & 0,73 & 82 \\
\hline Novembro & Linear & 459,83 & 163,36 & 0,73 & 82 \\
\hline Dezembro & Linear & 519,12 & 869,65 & 1,00 & 111 \\
\hline
\end{tabular}


$\mathrm{Na}$ Tabela 4, os valores do efeito pepita $\left(\mathrm{c}_{0}\right)$ são relativamente reduzidos, comparando-se com os valores encontrados por Siqueira et al. (2007) para o Estado vizinho (Minas Gerais), que variaram entre 6,11 e 1650. Tais valores indicam que a variabilidade espacial não detectada no processo de amostragem e que podem ser utilizados como indicador da precisão da amostragem (Siqueira et al., 2008). Uma opção para redução da variabilidade consiste no aumento do número de amostras (postos pluviométricos) (Vieira et al., 2010). Os valores de alcance encontrados no estudo foram relativamente altos, variando entre 54 e $111 \mathrm{~km}\left(0,49^{\circ}\right.$ e $\left.1,00^{\circ}\right)$, indicativos de alta variabilidade espacial. Todavia, são significativamente inferiores aos encontrados por Siqueira et al. (2007) (entre $1,73^{\circ}$ e $5,46^{\circ}$ ), para Minas Gerais.

A Figura 3 mostra os mapas contendo a variabilidade espacial da precipitação média mensal no Espírito Santo, obtidos utilizando-se os interpoladores com melhor desempenho estatístico calculado.

Verifica-se que as regiões montanhosas apresentaram as maiores precipitações médias mensais para os meses de janeiro, novembro e dezembro, com totais acumulados mensais superiores a $120 \mathrm{~mm}$ (Figura 3). Essa é uma característica marcante do Sudeste brasileiro (Sant'anna Neto, 2005), sendo que esses altos valores se devem ao fato de essas porções se encontrarem nas partes mais elevadas do Estado, sujeitas à entrada de ventos alísios e de massa de ar quente e úmido de origem marítima que, sob forte influência da topografia, provoca a ocorrência de chuvas orográficas nas vertentes a barlavento e a sotavento (Roe, 2005; Sant'anna Neto, 2005; Melo Júnior et al., 2006).

Na Figura 3, observa-se, ainda, a tendência de decréscimo da precipitação mensal no sentido SulNorte, mais nitidamente no período do verão, no qual a penetração ao anticiclone Polar Atlântico em contato com as massas tropicais provoca a formação de sistemas frontais, produtoras de cerca de dois terços da precipitação, segundo Sant'anna Neto (2005).

A região litorânea tende a apresentar as maiores precipitações mensais no inverno, o que se deve à atuação de frentes frias originárias do Sul (Moraes,
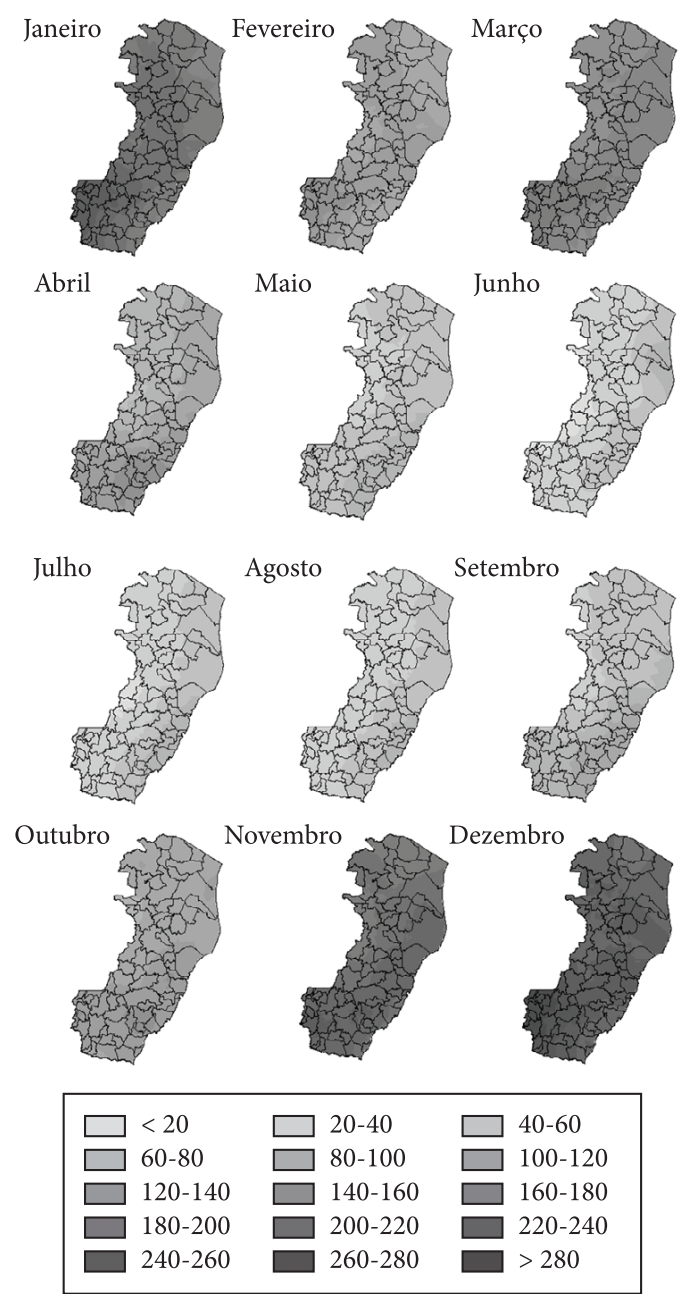

Figura 3. Mapas de variabilidade espacial das precipitações mensais (em milímetros) para o Espírito Santo.

Figure 3. Spatial variability maps of monthly rainfall (millimeters) at Espírito Santo.

2004) que, associadas à penetração dos ventos alísios (Sant’anna Neto, 2005) e à maritimidade, atuam como sistema formador da precipitação nessa porção do Espírito Santo.

As menores precipitações, invariavelmente, se concentram no Norte e no Nordeste do Estado, caracterizados por período chuvoso mais curto (Minuzzi et al., 2007) e por menores totais pluviométricos anuais, de período seco e de período chuvoso (Ribeiro, 2009; Castro et al., 2010). Tal fato deve-se à ação conjunta das condições climáticas associadas à topografia mais suave, com efeito orográfico menos pronunciado. Além disso, a 
região Serrana localizada mais a Sul/Sudoeste tem papel fundamental na distribuição das chuvas, ocasionando o fenômeno de sombra pluviométrica (Sant'anna Neto, 2005).

\section{CONCLUSÕES}

A Krigagem ordinária é o método de interpolação que apresenta as melhores estimativas da precipitação mensal no Espírito Santo.

O modelo linear de Krigagem ordinária é o de melhor desempenho para estimativas da precipitação mensal no Espírito Santo em 11 dos 12 meses.

As regiões Norte e Noroeste do Espírito Santo apresentam os menores totais mensais precipitados.

As regiões montanhosas e litorâneas apresentam os maiores totais precipitados no verão e inverno, respectivamente.

\section{STATUS DA SUBMISSÃO}

Recebido: 31/08/2011

Aceito:16/11/2011

Resumo publicado online: 17/11/2011

Artigo completo publicado: 22/12/2011

\section{AUTOR(ES) PARA CORRESPONDÊNCIA}

\section{Roberto Avelino Cecílio}

Departamento de Engenharia Florestal, Universidade Federal do Espírito Santo - UFES, Av. Carlos Lindemberg, 316, Centro, CEP 29550-000, Jerônimo Monteiro, ES, Brasil e-mail: roberto.cecilio@ufes.br

\section{REFERENNCIAS}

Akkala A, Devabhaktuni V, Kumar A. Interpolation techniques and associated software for environmental data. Environmental Progress \& Sustainable Energy 2010; 29(2):134-141. http://dx.doi.org/10.1002/ ep. 10455

Amorim RCF, Ribeiro A, Leite CC, Leal BG, Silva JBG. Avaliação do desempenho de dos métodos de espacialização da precipitação pluvial para o estado de Alagoas. Acta Scientiarum Technology 2008; 30(1):8791.
Barbosa FC, Teixeira AS, Gondim RS. Espacialização da evapotranspiração de referência e precipitação efetiva para estimativa das necessidades de irrigação na região do Baixo Jaguaribe-CE. Revista Ciência Agronômica 2005; 36(1):24-33.

Barbosa JPM. Utilização de método de interpolação para análise e espacialização de dados climáticos: O Sig como ferramenta. Caminhos da Geografia (UFU) 2006; 7(17):85-96.

Baú AL, Gomes BM, Queiroz MMF,Opazo MAU, Sampaio SC. Comportamento espacial da precipitação pluvial mensal provável da mesoregião oeste do estado do Paraná. Revista Irriga 2006; 11(2):150-168.

Blain GC, Pezzopane JRM, Pezzopane JEM, Bonomo R. Índice padronizado de precipitação aplicado às condições de seca no Estado do Espírito Santo. Revista Brasileira de Engenharia Agrícola e Ambiental 2010; 14(10):1067-1073.

Camargo ÂP, Sentelhas PC. Avaliação do desempenho de diferentes métodos de estimativa da evapotranspiração potencial no estado de São Paulo, Brasil. Revista Brasileira de Agrometeorologia 1997; 5(1):89-97.

Caruso C, Quarta F. Interpolation methods comparison. Computers Mathematical Application 1998; 35:109-126. http://dx.doi.org/10.1016/S0898-1221(98)00101-1

Carvalho JRP, Assad ED. Análise espacial da precipitação pluviométrica no estado de São Paulo: Comparação de métodos de interpolação. Engenharia Agrícola 2005; 25(2):377-384. http://dx.doi.org/10.1590/ S0100-69162005000200011

Castro FS, Pezzopane JEM, Cecílio RA, Pezzopane JRM, Xavier AC. Avaliação do desempenho dos diferentes métodos de interpoladores para parâmetros do balanço hídrico climatológico. Revista Brasileira de Engenharia Agrícola e Ambiental 2010; 14:871-880.

Fabry F. Meteorological value of ground target measurements by radar. Journal of Atmospheric and Oceanic Technology 2004; 21:560-573. http://dx.doi. org/10.1175/1520-0426(2004)021\%3C0560:MVOGTM \%3E2.0.CO;2

Falivene O, Cabrera L, Tolosana-Delgado R, Sáez A. Interpolation algorithm ranking using cross-validation and the role of smoothing effect. A coal zone example. Computers \& Geosciences 2010; 36(4):512-519. http:// dx.doi.org/10.1016/j.cageo.2009.09.015

Keller Filho T, Assad ED, Lima PRSR. Regiões pluviometricamente homogêneas no Brasil. Pesquisa Agropecuária Brasileira 2005; 40(4):311-322.

Legates DR, McCabe Junior GJ. Evaluating the use of "goodness-of-fit" measures in hydrologic and hydroclimatic model validation. Water Resources Research 1999; 35(1):233-241. http://dx.doi. org/10.1029/1998WR900018 
Lu GY, Wong DW. An adaptive inverse-distance weighting spatial interpolation technique. Computers \& Geosciences 2008; 34:1044-1055. http://dx.doi. org/10.1016/j.cageo.2007.07.010

MathWorks. MATLAB software. Version 6.0. Natick: The MathWorks, Inc.; 2000.

Melo Júnior JCF, Sediyama GC, Ferreira PA, Leal BG. Determinação de regiões homogêneas quanto à distribuição de freqüência de chuvas no leste do Estado de Minas Gerais. Revista Brasileira de Engenharia Agrícola e Ambiental 2006; 10(2):408-416. http://dx.doi. org/10.1590/S1415-43662006000200023

Minuzzi RB, Sediyama GC, Barbosa EM, Melo Júnior JCF. Climatologia do comportamento do período chuvoso da região sudeste do Brasil. Revista Brasileira de Meteorologia 2007; 22(3):338-344.

Miranda F, Freitas S, Faggion P. Integração e interpolação de dados de anomalias ar livre utilizandose a técnica de RNA e krigagem. Boletim de Ciências Geodésicas 2009; 15(3):428-433.

Moraes C. Geografia do Espírito Santo. Vitória:Instituto Histórico e Geográfico do Espírito Santo; 2004.

Price DT, McKenney DW, Nalder IA, Hutchinson MF, Kesteven JL. A comparison of two statistical methods for spatial interpolation of Canadian monthly mean climate data. Agriculturaland Forest Meteorology 2000; 101:8194. http://dx.doi.org/10.1016/S0168-1923(99)00169-0

Quadro M, Machado LHR, Calbete S, Batista N, Oliveira GS. Climatologia e Precipitação e Temperatura no período de 1986 a 1996. Climanálise 1996; 1:90.

Ribeiro CAD. Delimitação de zonas agroclimáticas para cultura do eucalipto no norte do Espírito Santo e sul da Bahia [dissertação].Alegre: Universidade Federal do Espírito Santo; 2009.

Robinson TP, Metternicht G. Testing the performance of spatial interpolation techniques for mapping soil properties. Computers and Electronics in Agriculture 2006; 97-108. http://dx.doi.org/10.1016/j. compag.2005.07.003

Roe GH. Orographic precipitation. Annual Review of Earth and Planetary Sciences 2005; 33:645-671. http:// dx.doi.org/10.1146/annurev.earth.33.092203.122541

Sant’anna Neto JL. Decálogo da climatologia do Sudeste Brasileiro. Revista Brasileira de Climatologia 2005; 1(1):43-60.

Silva JW, Guimarães EC, Tavares M. Variabilidade temporal da precipitação mensal e anual na estação climatológica de Uberaba-MG. Ciência \& Agrotecnologia 2003; 27(3):665-674. http://dx.doi. org/10.1590/S1413-70542003000300023
Silva VPR, Pereira ERR, Azevedo PA, Sousa FAS, Sousa FI. Análise da pluviometria e dias chuvosos na região Nordeste do Brasil. Revista Brasileira de Engenharia Agrícola e Ambiental 2011; 15(2):131-138. http://dx.doi. org/10.1590/S1415-43662011000200004

Siqueira HR, Alves GF, Guimarães EC. Comportamento da precipitação pluviométrica mensal do estado de Minas Gerais: análise espacial e temporal. Horizonte Científico 2007; 1(1):1-21.

Siqueira JDP, Lisboa RS, Ferreira AM, Souza MFR, Araújo E, Lisbão Júnior L, Siqueira, M.M. Estudo ambiental para os programas de fomento florestal da Aracruz Celulose S.A. e extensão florestal do governo do estado do Espírito Santo. Floresta 2004; (especial):3-67.

Siqueira GM, Vieira SR, Ceddia MB. Variabilidade de atributos físicos do solo determinados por métodos diversos. Bragantia 2008; 67(1):203-211. http://dx.doi. org/10.1590/S0006-87052008000100025

Sivapragasam C, Arun VM, Giridhar D.A simple approach for improving spatial interpolation of rainfall using ANN. Meteorology and Atmospheric Physics 2010; 109(1-2). http://dx.doi.org/10.1007/ s00703-010-0090-Z

Tao T, Chocat B, Liu S, Xin K. Uncertainty analysis of interpolation methods in rainfall spatial distribution-a case of small catchment in Lyon. Journal of Water Resource and Protection 2009; 2:136-144. http://dx.doi. org/10.4236/jwarp.2009.12018

Teegavarapu RSV, Chandramouli V. Improved weighting methods, deterministic and stochastic datadriven models for estimation of missing precipitation records.Journal of Hydrology 2005; 312:191-206. http:// dx.doi.org/10.1016/j.jhydrol.2005.02.015

Vianello RL, Alves, AR. Meteorologia básica e aplicações. Viçosa: UFV; 2000. 448p.

Vieira SR. Geoestatística em estudos de variabilidade espacial do solo. In: Novais RF, Alvares VVH, Schaeffer CEGR, editors. Tópicos em ciência do solo. Viçosa: Sociedade Brasileira de Ciência do Solo; 2000. p.1-54.

Vieira SR, Camargo MBP, Siqueira G. Análise espacial da temperatura e precipitação média anual no estado de São Paulo (Brasil). Revista Brasileira de Engenharia de Biossistemas 2010; 3(2):87-97.

Viola MR, Mello CR, Pinto DBF, Mello JM, Ávila LF. Métodos de interpolação espacial para o mapeamento da precipitação pluvial. Revista Brasileira de Engenharia Agrícola e Ambiental 2010; 14(9):970-978. http://dx.doi. org/10.1590/S1415-43662010000900009

Xavier AC, Cecílio RA, Lima JSS. Módulos em MATLAB para interpolação espacial pelo método de krigagem ordinária e do inverso da distância. Revista Brasileira de Cartografia 2010;62:67-76. 